\title{
Zebrin-Immunopositive and -Immunonegative Stripe Pairs Represent Functional Units in the Pigeon Vestibulocerebellum
}

\author{
David J. Graham ${ }^{1}$ and Douglas R. Wylie ${ }^{1,2}$ \\ ${ }^{1}$ Centre for Neuroscience, ${ }^{2}$ Department of Psychology, University of Alberta, Edmonton, Alberta T6G 2E9, Canada
}

\begin{abstract}
The cerebellum is a site of complex sensorimotor integration and contains up to $80 \%$ of the neurons in the brain, yet comparatively little is known about the organization of sensorimotor systems within the cerebellum. It is known that afferent projections and Purkinje cell (PC) response properties are organized into sagittal "zones" in the cerebellum. Moreover, the isoenzyme aldolase C [also known as zebrin II (ZII)] is heterogeneously expressed in cerebellar PCs such that there are sagittal stripes of PCs with high expression (ZII+) interdigitated with stripes of little or no expression (ZII-). In the present study, we show how the ZII stripes in folium IXcd of the vestibulocerebellum in pigeons are related to response properties of PCs. IXcd consists of seven pairs of ZII $+/-$ stripes denoted P1 $+/-$ (medial) to P7+/- (lateral). Electrophysiological studies have shown that vestibulocerebellar PCs respond to particular patterns of optic flow resulting from self-motion in three-dimensional space. In our study, we recorded optic flow preferences from PCs in IXcd, marked recording locations with injections of fluorescent tracer, and subsequently immunoreacted coronal sections for ZII. We found that the PCs within a ZII $+/$ - stripe pair all responded best to the same pattern of optic flow. That is, a ZII + / - stripe pair forms a functional unit in the cerebellum. This is the first demonstration that the function of PCs is associated with ZII stripes across the mediolateral extent of an entire folium.
\end{abstract}

\section{Introduction}

One of the fundamental questions in neuroscience considers how sensorimotor information is organized in the brain. In the cerebral cortex, the general principles are well established: columnar organization (Hubel and Wiesel, 1962; Mountcastle, 1997), modules (Goldman-Rakic, 1984; Blasdel and Salama, 1986), hierarchical organization (Felleman and Van Essen, 1991), and streams (Ungerleider and Mishkin, 1982; Goodale and Milner, 1992). The cerebellum is a site of extensive sensorimotor organization, and although the cerebellum contains up to $80 \%$ of the neurons in the brain (Herculano-Houzel, 2010), comparatively less is known about its organization of sensorimotor systems.

It is established that the cerebellum consists of sagittal "zones" (Voogd and Bigare, 1980). This is apparent in the pattern of afferent inputs, projection patterns of Purkinje cells (PCs), and PC response properties (Voogd, 1967; Ekerot and Larson, 1973; Andersson and Oscarsson, 1978; Llinás and Sasaki, 1989; De Zeeuw et al., 1994; Wu et al., 1999; Ruigrok, 2003). The expres-

Received Jan. 13, 2012; revised July 3, 2012; accepted July 23, 2012.

Author contributions: D.J.G. and D.R.W. designed research; D.J.G. and D.R.W. performed research; D.J.G. and D.R.W. analyzed data; D.J.G. and D.R.W. wrote the paper.

This work was supported by funding to D.R.W. from the Canadian Institute for Health Research and the Canadian Foundation for Innovation. D.R.W. was supported by funding from the Canada Research Chairs Program. D.J.G. was supported by graduate scholarships from Natural Sciences and Engineering Research Council of Canada. We thank Dr. Richard Hawkes from the University of Calgary for kindly providing us with the Zebrin II antibody.

Correspondence should be addressed to Douglas R. Wylie, Department of Psychology, University of Alberta, Edmonton, Alberta T6G 2E9, Canada. E-mail: dwylie@ualberta.ca.

DOI:10.1523/JNEUROSCI.0197-12.2012

Copyright $\odot 2012$ the authors $\quad 0270-6474 / 12 / 3212769-11 \$ 15.00 / 0$ sion of several molecular markers is also parasagittally organized (Hawkes and Gravel, 1991; Hawkes, 1992), including zebrin II (ZII) (aldolase C) (Brochu et al., 1990; Ahn et al., 1994; Hawkes and Herrup, 1995). ZII is expressed in PCs such that there are sagittal stripes of high expression $(\mathrm{ZII}+)$ interdigitated with stripes of little expression $(\mathrm{ZII}-)$. The spatial pattern and number of ZII stripes is highly conserved in the cerebella of birds and mammals (Pakan et al., 2007; Iwaniuk et al., 2009; Marzban and Hawkes, 2011), indicating that ZII may be critical for cerebellar function.

Much research has examined how the ZII stripes are related to efferent and afferent projections (Voogd et al., 2003; Sugihara and Shinoda, 2004, 2007; Voogd and Ruigrok, 2004; Sugihara and Quy, 2007; Sugihara, 2011). Fewer studies have attempted to relate the sagittal ZII stripes to PC function (Gao et al., 2006; Sugihara et al., 2007; Mostofi et al., 2010). In this regard, our laboratory has been investigating the pigeon vestibulocerebellum (VbC) (folia IXcd and X) where complex spike activity (CSA) of PCs responds best to optic flow stimuli (Graf et al., 1988; Wylie and Frost, 1999). PCs in the VbC respond to optic flow resulting from either self-rotation (flocculus) or self-translation (ventral uvula and nodulus) (Fig. $1 A$ ). In the flocculus, CSA responds to self-rotation about the vertical axis ("rVA"), or a horizontal axis ("rHA"). We showed that rVA neurons are localized to the $\mathrm{P} 4+/-$ and $\mathrm{P} 6+/-\mathrm{ZII}$ stripes in IXcd, whereas rHA neurons are localized to the P5+/- and P7+/- ZII stripes (Pakan et al., 2011), suggesting that a functional zone spans a ZII $+/$ - stripe pair. Whether this applies generally to the rest of the cerebellum remains uncertain. In this study, we recorded CSA in the uvula to 
patterns of optic flow, marked the recording locations, and localized the recording sites to ZII stripes. Our data show how the optic flow zones are correlated with ZII heterogeneity across the entire mediolateral extent of a folium. Moreover, we affirm that a ZII+/ - stripe pair represents a functional unit in the pigeon cerebellum.

\section{Materials and Methods}

Surgery and electrophysiological recording procedures in the ventral uvula and nodulus. The methods reported herein conformed to the guidelines established by the Canadian Council on Animal Care and were approved by the Biosciences Animal Care and Use Committee at the University of Alberta. Procedures were optimized for minimizing the number of animals used. Twenty-four Silver King and Homing pigeons (Columba livia) of either sex, obtained from a local supplier, were anesthetized by an intramuscular injection of a ketamine $(65 \mathrm{mg} /$ $\mathrm{kg}) /$ xylazine $(8 \mathrm{mg} / \mathrm{kg})$ mixture. Supplemental doses were administered as necessary. Animals were placed in a stereotaxic device with pigeon ear bars and a beak bar adapter so that the orientation of the skull conformed to the atlas of Karten and Hodos (1967). The dorsal surface of the left cerebellum was accessed by removing the bone above folia VI and VII, as the medial $\mathrm{VbC}$ (folia IXcd and X) lies below these folia. Extracellular single-unit recordings were then made using glass micropipettes filled with $2 \mathrm{M}$ $\mathrm{NaCl}$ (tip diameters of 3-5 $\mu \mathrm{m}$ ). Electrodes were advanced through folia VI-X using an hydraulic microdrive (Frederick Haer). Raw signals were amplified, filtered $(10-2000 \mathrm{~Hz})$, and digitized by a data analysis system (Cambridge Electronic Design 1401plus) at a sampling rate of $16,667 \mathrm{~Hz}$. Using the spike sorting program in Spike2 (Cambridge Electronic Design), complex and simple spikes of PCs were sorted off-line.

We determined the optic flow preferences of neurons as described in previous studies (Winship and Wylie, 2001, 2003; Wylie et al., 2003a,b; Pakan et al., 2011). Briefly, visual responsiveness of CSA was first determined with a large handheld stimulus, which consisted of visual noise. By moving this stimulus in different areas of the panoramic binocular visual field, the optic flow preference of each unit was qualitatively determined. Visual stimuli were then backprojected onto a screen measuring $90 \times 75^{\circ}$ (width by height) that was positioned in the frontal visual field (from $45^{\circ}$ ipsilateral to $45^{\circ}$ contralateral azimuth), the contralateral visual field (from $45^{\circ}$ contralateral azimuth to $135^{\circ}$ contralateral azimuth), or the ipsilateral visual field (from $45^{\circ}$ ipsilateral azimuth to $135^{\circ}$ ipsilateral azimuth; see Fig. $3 A$ ). We refer to these as the frontal, contralateral, and ipsilateral visual fields, respectively. The stimuli consisted of drifting sine or square wave gratings of an effective spatial and temporal frequency $(0.125-0.5$ cycles per degree, $0.125-0.5 \mathrm{~Hz}$ ), generated by a VSGThree visual stimulus generator (Cambridge Research Systems). Direction tuning was established by measuring the responses to motion in eight directions, $45^{\circ}$ apart (see Fig. $3 B$ ). Responses were averaged over at least three sweeps, where each sweep consisted of $5 \mathrm{~s}$ of motion in one direction, a $4 \mathrm{~s}$ pause, and $5 \mathrm{~s}$ of motion in the opposite direction, followed by a $7 \mathrm{~s}$ pause (see Fig. $3 C, D$ ). Polar plots of directional tuning (see Fig. $3 E-I$ ) were constructed using
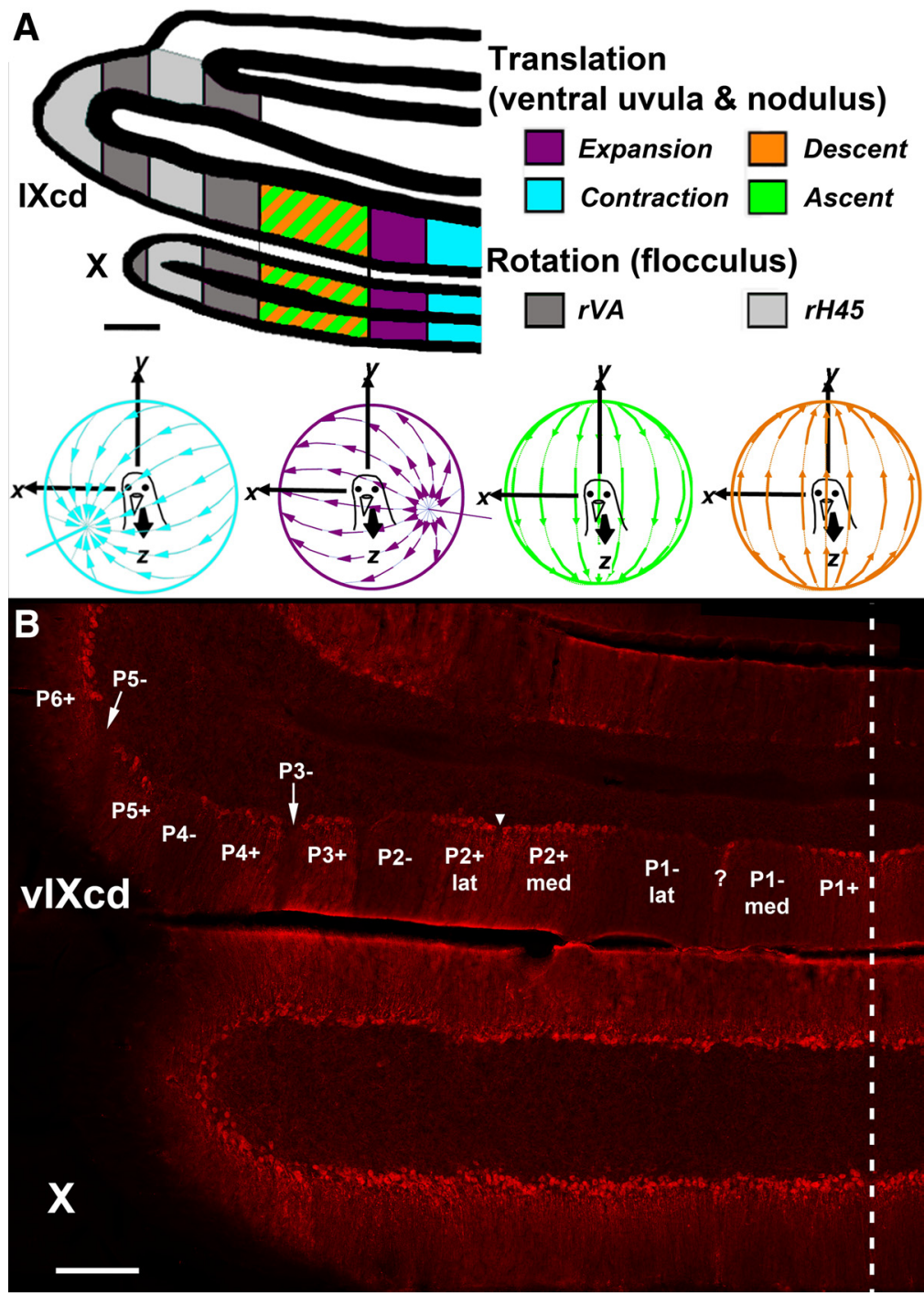

Figure 1. Zll organization and electrophysiological zones in the medial VbC (i.e., ventral uvula and nodulus) of pigeons. A shows the four functional classes of Purkinje cells responsive to patterns of optic flow resulting from self-translation in three-dimensional space: contraction (light blue), expansion (purple), ascent (green), and descent (orange) [based on the study by Wylie and Frost ( In the lateral VbC (i.e., flocculus), Purkinje cells are responsive to patterns of optic flow resulting from self-rotation about either the vertical axis ( $\mathrm{rVA}$ ) or an horizontal axes oriented $45^{\circ}$ to the midline ( $\left.\mathrm{rH} 45\right)$ as indicated by gray shading (Wylie and Frost, Wylie et al., 1993). B shows a coronal section through folia IXcd and Ximmunoreacted for Zll. The Zll stripes are numbered PI ventral IXcd as well as P6 +.P6- P7+, and P7 - are found more rostrally (Pakan et al., 2007).P1 - is divided into medial and lateral portions by a small satellite immunopositive band one to two Purkinje cells wide in the middle of P1 - denoted "?." P2+ is divided into medial and lateral portions by a small immunonegative "notch" in the middle of $\mathrm{P} 2+$ (see inverted triangle). Folium $X$ does not have ZII stripes, as all Purkinje cells are ZII +. Scale bars: $A, 500 \mu \mathrm{m} ; \boldsymbol{B}, 300 \mu \mathrm{m}$.

SigmaPlot (Systat Software). With these procedures, "contraction," "expansion," "ascent," and "descent" neurons are easy to distinguish (Winship and Wylie, 2006): in the left VbC, contraction and expansion neurons show maximum excitation/(inhibition) to rightward/(leftward) motion and little modulation to vertical motion in the frontal visual field (see Fig. $3 E, F$ ). Contraction neurons are differentiated from expansion neurons in the ipsilateral and contralateral fields, where contraction neurons show maximum excitation/(inhibition) to temporal-to-nasal (T$\mathrm{N}) /(\mathrm{N}-\mathrm{T})$ motion, while expansion neurons show maximum excitation/ (inhibition) to N-T/(T-N) motion (see Fig. 3E,F). rVA neurons in the flocculus also show maximum excitation/(inhibition) to rightward/(leftward) motion in the frontal visual field. However, rVA neurons are differentiated from contraction and expansion neurons as they show maximum excitation/(inhibition) to T-N/(N-T) motion in the ipsilateral field, and maximum excitation/(inhibition) to $\mathrm{N}-\mathrm{T} /(\mathrm{T}-\mathrm{N})$ motion in the 

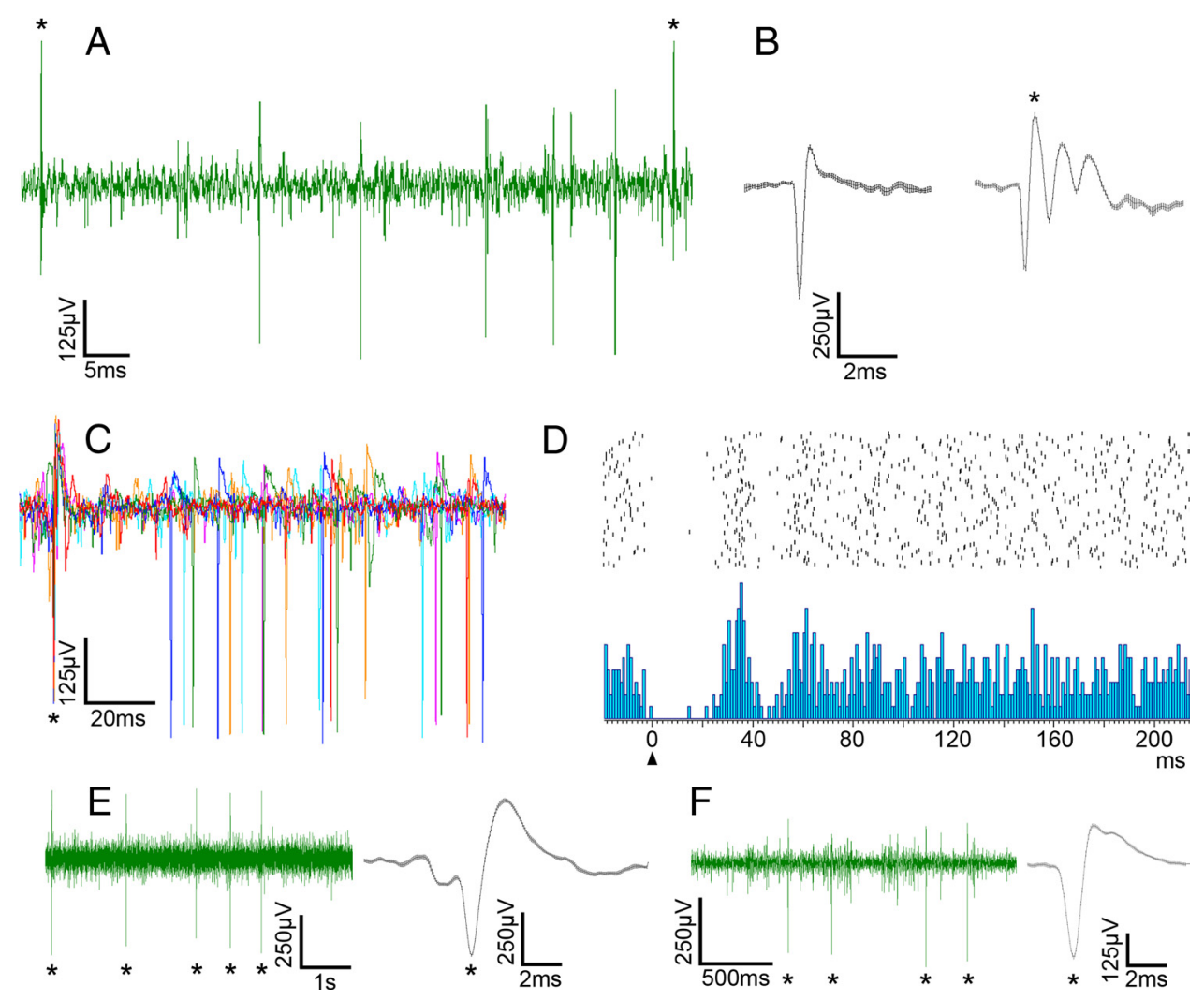

Figure 2. Identification of complex and simple spike activity in the ventral uvula and nodulus. $A$ shows the recording of $P C$ activity. Both CSA and SSA can be seen. $B$ shows the average waveform of a simple spike and a complex spike from a PC. Both waveforms were over 20 consecutive spikes. The error bars at each point are the SEM. C shows six superimposed traces to illustrate the SSA pause following a complex spike. These were synchronized to the initial positive peak of the complex spikes. D shows a peri-complex spike time histogram (below; 102 repetitions; bin width, $1 \mathrm{~ms}$ ) showing a pause-facilitation of the simple spike after the onset (arrowhead) of a complex spike, and a raster diagram of all the simple spikes that contributed to the histogram (above). $\boldsymbol{E}$ and $\boldsymbol{F}$ show recording of CSA in the molecular layer. For $\boldsymbol{E}$, a standard recording electrode was used (glass micropipette with $2 \mathrm{M} \mathrm{NaCl}$; tip diameter, $\sim 5 \mu \mathrm{m}$ ). For $\boldsymbol{F}$, a standard injection electrode was used (glass micropipette filled with $1 \%$ (TB-Alexa Fluor 594 in $0.1 \mathrm{~m} \mathrm{~PB}$; tip diameter $\sim 25 \mu \mathrm{m}$ ). The average of 20 consecutive complex spikes ( \pm SEM) is shown for each. The asterisks indicate complex spikes.

contralateral field (see Fig. 3I). Descent neurons show maximum excitation/(inhibition) to upward/(downward) motion in all visual fields (see Fig. $3 G$ ), and ascent neurons show maximum excitation/(inhibition) to downward/(upward) motion (see Fig. $3 H$ ). Both ascent and descent neurons show little modulation to horizontal motion (see Fig. 3G,H) (Wylie et al., 2003b). A preferred direction (PD) for each unit was determined by calculating the mean vector as follows:

$$
\mathrm{PD}=\tan ^{-1}\left(\frac{\sum_{n}\left(\mathrm{FR}_{n} \times \sin \theta_{n}\right)}{\sum_{n}\left(\mathrm{FR}_{n} \times \cos \theta_{n}\right)}\right),
$$

where FR represents firing rate and $n$ represents the eight directions of motion. A modulation index (MI) was also calculated for each unit as follows:

$$
\mathrm{MI}=\left(\frac{\max \text { response }-\min \text { response }}{\max \text { response }+\min \text { response }}\right) .
$$

Thus, if the maximum response was twice that of the minimum response, $\mathrm{MI}=0.33$.

Several electrode penetrations were made to map out the locations of the different translational optic flow zones in the medial VbC. To mark some of the recording sites, and thus reconstruct the recording locations, we made injections of red and green fluorescent tracers at locations in the ventral uvula and nodulus from which we had recorded CSA to visual stimuli (see Fig. 5A-C). For accurate placement of the injection electrodes and reconstruction of the electrode tracts to localize each recorded cell to a specific ZII zone, we relied on stereotaxic coordinates. The injection electrodes were micropipettes (tip diameter, $20-30 \mu \mathrm{m}$ ) containing $1 \%$ cholera toxin subunit $\mathrm{B}$
(CTB) [either CTB-Alexa Fluor 488 (green) or 594 (red) conjugate (Invitrogen)]. CTB was iontophoresed for $5-10 \mathrm{~min}(+4 \mu \mathrm{A} ; 7 \mathrm{~s}$ on, $7 \mathrm{~s}$ off). Injections of CTB were made to both mark a recording location and serve as a reference point to which all other recordings within that mediolateral plane could be stereotaxically measured and located. The advantage of using CTB was that we could reliably record CSA with the injecting electrode to confirm the response type of the neuron (see Fig. 2 F) (Pakan et al., 2011). Note that the CTB injections were only used to mark the locations of recording sites. Because of the lengths of the recording sessions, which lasted a minimum of $12 \mathrm{~h}$, we were advised by the Biosciences Animal Care and Use Committee at the University of Alberta not to allow the animals to recover after surgery. Thus, CTB was not used as a retrograde tracer.

At the end of the experiments, the pigeons were deeply anesthetized with an overdose of sodium pentobarbital $(100 \mathrm{mg} / \mathrm{kg})$ and immediately transcardially perfused with PBS $(0.9 \% \mathrm{NaCl}, 0.1 \mathrm{M}$ phosphate buffer $)$ followed by $4 \%$ paraformaldehyde in $0.1 \mathrm{M}$ PBS, $\mathrm{pH}$ 7.4. The brain was extracted from the skull and immersed in paraformaldehyde for $7 \mathrm{~d}$ at $4^{\circ} \mathrm{C}$. The brain was then embedded in gelatin and cryoprotected by placing it in 30\% sucrose in $0.1 \mathrm{M}$ PBS until it sank. Using a microtome, frozen serial sections through the cerebellum in the coronal plane were cut (40 $\mu \mathrm{m}$ thick) and collated into two series.

Zebrin II immunohistochemistry. ZII expression was visualized using established immunohistochemical techniques described previously ( $\mathrm{Pa}$ kan et al., 2007). Briefly, sections were rinsed thoroughly in $0.1 \mathrm{M}$ PBS and blocked with 10\% normal donkey serum (Jackson ImmunoResearch Laboratories) and $0.4 \%$ Triton X-100 in PBS for $1 \mathrm{~h}$. Tissue was then incubated in PBS containing $0.1 \%$ Triton X-100 and the primary antibody, mouse monoclonal anti-zebrin II (kindly provided by Richard 


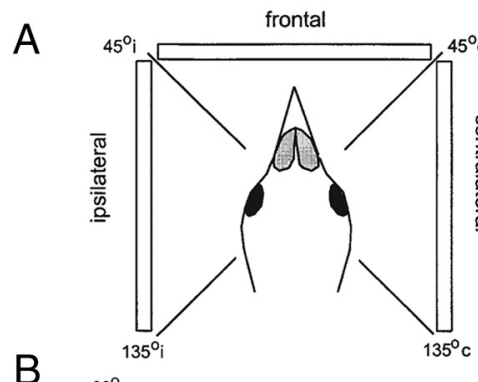

B
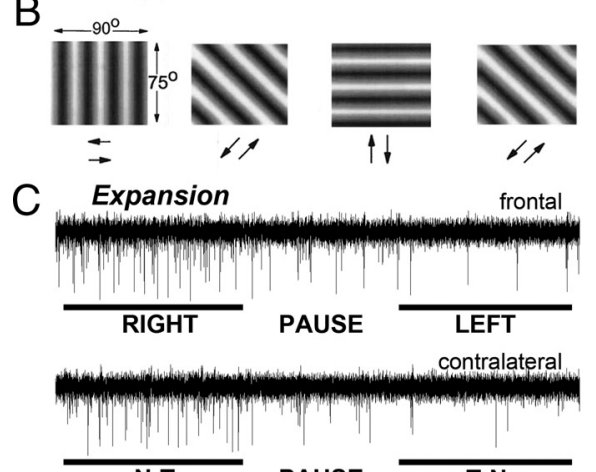

N-T

PAUSE

T-N

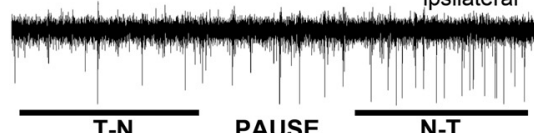

T-N

$\mathrm{D}$

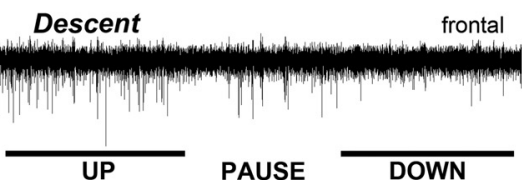

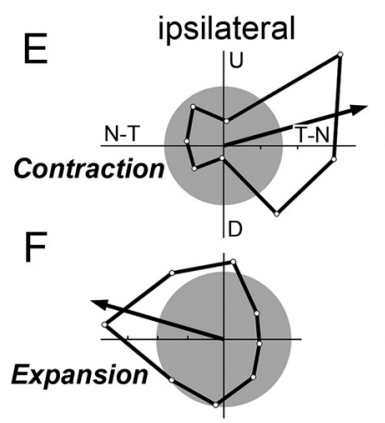
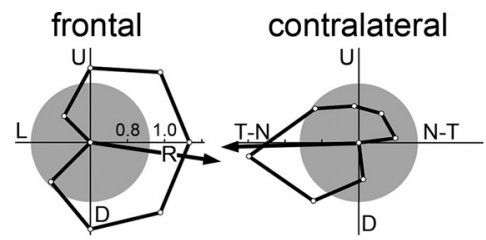

G
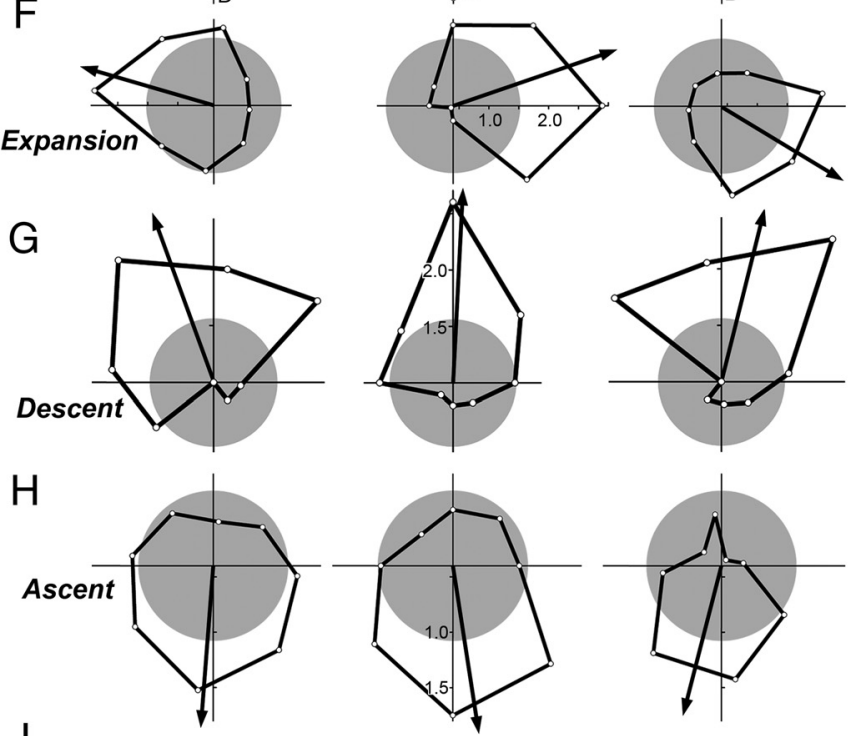

I
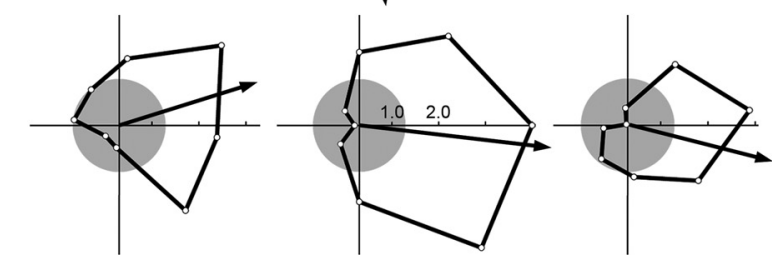

Figure 3. CSA of Purkinje cells in the vestibulocerebellum in response to moving large-field stimuli presented in different regions of the visual field. $A$, The screen was positioned at one of three locations relative to the bird: the ipsilateral, contralateral, and frontal regions (assuming recordings were made in the left VbC). $B$ shows the four grating orientations used to determine tuning curves in each location. Drifting sine and square wave gratings were backprojected onto the screen that measured $90 \times 75^{\circ}$ (width by height). Each grating moved in both directions, perpendicular to the orientation of the grating. Direction tuning curves were constructed by plotting the response as a function of the eight directions of motion ( $\boldsymbol{E}-\boldsymbol{I})$. $(\boldsymbol{C}$ shows a recording of the response of an expansion cell to a single sweep of horizontal motion in the frontal, contralateral, and ipsilateral fields. D shows the recording of a descent cell to a single sweep of vertical motion in the frontal field. Each sweep consisted of $5 \mathrm{~s}$ of motion in one direction, followed by a $4 \mathrm{~s}$ pause, and then $5 \mathrm{~s}$ of motion in the opposite direction. $\boldsymbol{E}$-I show direction tuning curves of contraction $(\boldsymbol{E})$, expansion $(\boldsymbol{F})$, descent $(\boldsymbol{G})$, ascent $(\boldsymbol{H})$, and rVA $(\boldsymbol{I})$ neurons for each of the three regions of the visual field (ipsilateral, frontal, contralateral). The tuning curves plot the firing rate as a function of direction of motion in polar coordinates. The gray circles represent the spontaneous rates, and the arrows indicate the preferred direction (i.e., the orientation of the mean vector). U, D, L, R, Upward, downward, leftward, and rightward motion. N-T, T-N, Nasal-to-temporal and temporal-to-nasal motion, respectively.

Hawkes, University of Calgary, Calgary, Alberta, Canada) (Brochu et al., $1990)$ for $5 \mathrm{~d}$ at $4^{\circ} \mathrm{C}$. Sections were then rinsed several times in PBS, and sections were then incubated in a fluorescent secondary antibody. Because recording sites were marked with injections of green and red tracer in the same animal, alternate series were visualized with Dylite 594 (red)or Dylite 488 (green)-conjugated donkey anti-mouse antibody (Jackson ImmunoResearch Laboratories; diluted 1:100 in PBS, 2.5\% normal donkey serum, and $0.4 \%$ Triton $\mathrm{X}-100)$ for $2 \mathrm{~h}$ at room temperature. The tissue was then rinsed several times in PBS and mounted onto gelatinized slides for viewing.

Microscopy and image analysis. Sections were viewed with a compound light microscope (Leica DMRE) equipped with the appropriate fluorescence filters (rhodamine and FITC). Images were acquired using a Retiga EXi FAST Cooled Mono 12-bit camera (QImaging) and analyzed with Openlab imaging software (Improvision). Adobe Photoshop was used to adjust for brightness and contrast.

\section{Results}

Electrophysiological recordings in the ventral uvula and nodulus

The results presented are based on experiments in 24 animals. Data were obtained from 125 PCs and representative recordings are shown in Figure 2. In 22 instances, we recorded at the PC layer where CSA and simple spike activity (SSA) are apparent (Fig. $2 A-C)$. Here, the complex spikes can be easily distinguished from the simple spikes based on both the polarity and the shape of the waveform (Fig. $2 A, B$ ). The characteristic pause in SSA following a complex spike was observed (Granit and Phillips, 1956; Bloedel and Roberts, 1971) (Fig. 2C,D). As we have shown previously (Wylie et al., 1993), SSA was not modulated by the visual stimuli. This may be due to the fact that a ketamine-anesthetized preparation was used (Sato et al., 1993).

For most units $(n=103)$, and as is typical practice, we recorded CSA in the molecular layer where the CSA is easier to isolate (Fig. $2 E, F$ ). Here, single complex spike units are easy to isolate based on amplitude alone $(n=70)$. For the remaining units, we relied on the spike sorting option. At 27 of these recording sites, we used the spike sort to isolate one unit. At each of three other recording sites, we isolated two units from the electrophysiological signal with the spike sort. The recordings were as reliable with injection electrodes (Fig. $2 F$ ) as they were with the recording 

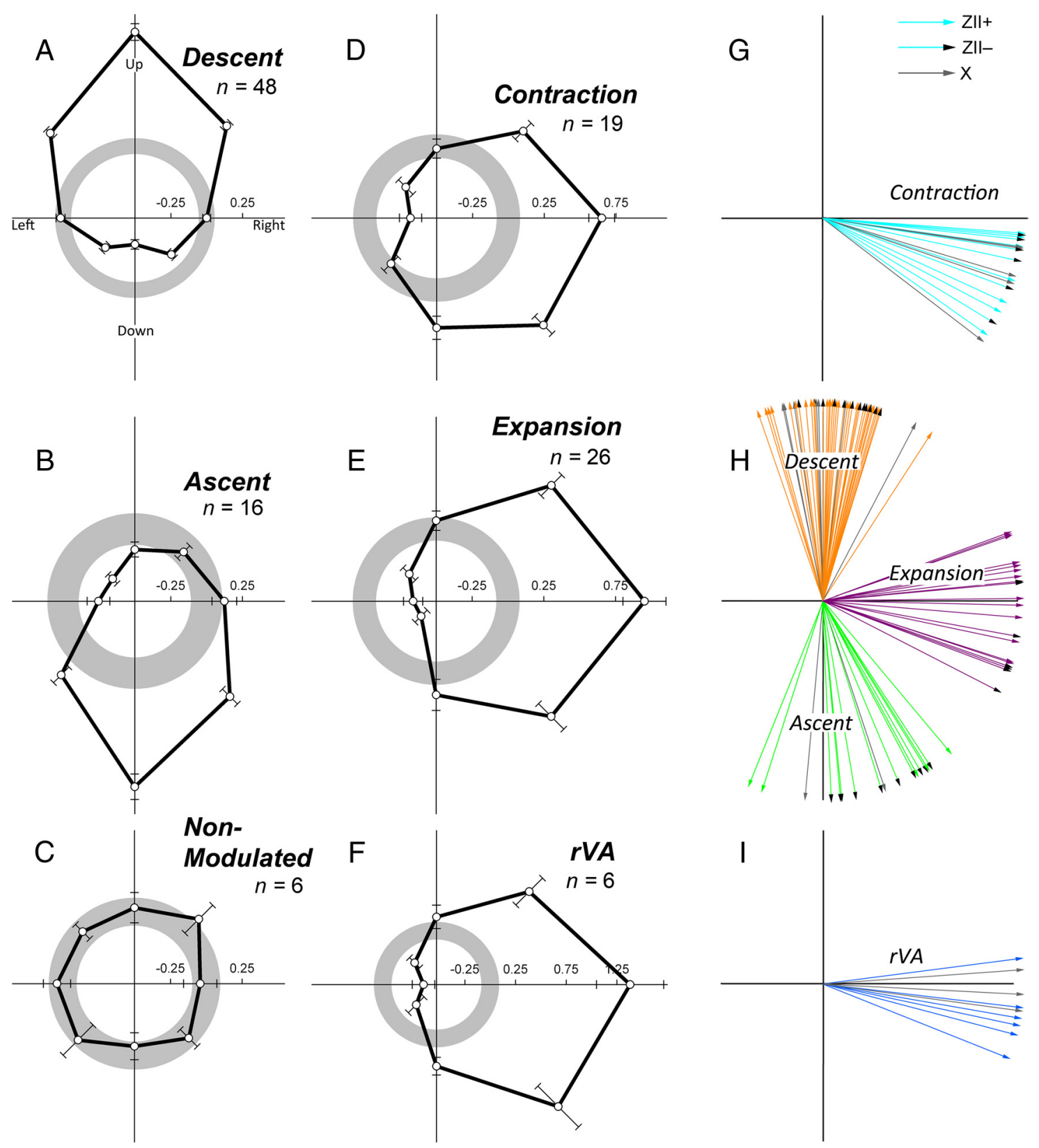

Figure 4. Average direction tuning curves and preferred direction of visual motion of Purkinje cells in the ventral uvula and nodulus. $\boldsymbol{A}-\boldsymbol{F}$ shows the average direction tuning curves, as measured in the frontal field, for all cell types encountered (descent, ascent, nonmodulated, contraction, expansion, rVA). These were averaged after subtracting the spontaneous rate (SR) from the firing rate in each direction from a given cell type. Average firing rate was plotted as a function of direction of motion in polar coordinates, and the error bars represent \pm 1 SEM. The gray circles indicate the average spontaneous rate (set to 0$) \pm 1$ SEM. G-I show the distributions of the preferred directions of all contraction, expansion, descent, ascent, and rVA cells by calculating the mean vector (for details, see Materials and Methods). For each of $\mathbf{G}-\mathbf{I}$, an arrow indicates the preferred direction of individual cells in a Zll + zone (solid, colored arrows), a Zll - zone (colored arrows with a black arrowhead), and folium X (solid, gray arrows).

electrodes (Fig. 2E). Across all 125 units, the average spontaneous firing rate was 1.18 spikes/s $( \pm 0.33 \mathrm{SD})$.

\section{Directional tuning}

The CSA of PCs showed either significant directional tuning, and were identified as contraction $(n=19)$, expansion $(n=26)$, ascent $(n=16)$, descent $(n=48)$, rVA $(n=10)$, or were not modulated by visual motion stimuli (NM) $(n=6)$. In Figure $3 C$, CSA recordings are shown for horizontal motion in the three fields for an expansion cell. Note the excitation (inhibition) to motion in the rightward (leftward) direction in the frontal field (top) and the excitation (inhibition) to N-T (T-N) motion in both the contralateral (middle) and ipsilateral (bottom) fields. Similarly in Figure 3D, a recording to vertical motion in the frontal field is shown for a descent cell. Note the excitation (inhibition) to upward (downward) motion. Representative directional tuning curves to large-field motion in the ipsilateral, frontal, and contralateral fields are shown for single units of the different cell types in Figure $3 E-I$. Firing rate is plotted as a function of direction, and the preferred directions, determined from the mean vector, are indicated by the arrows. Figure $4 A-F$ shows average normalized tuning curves for each of the cell types to visual motion stimuli in the frontal visual field. In Figure $4 G-I$, the distribution of the PDs for each of the cell types are shown. The average 

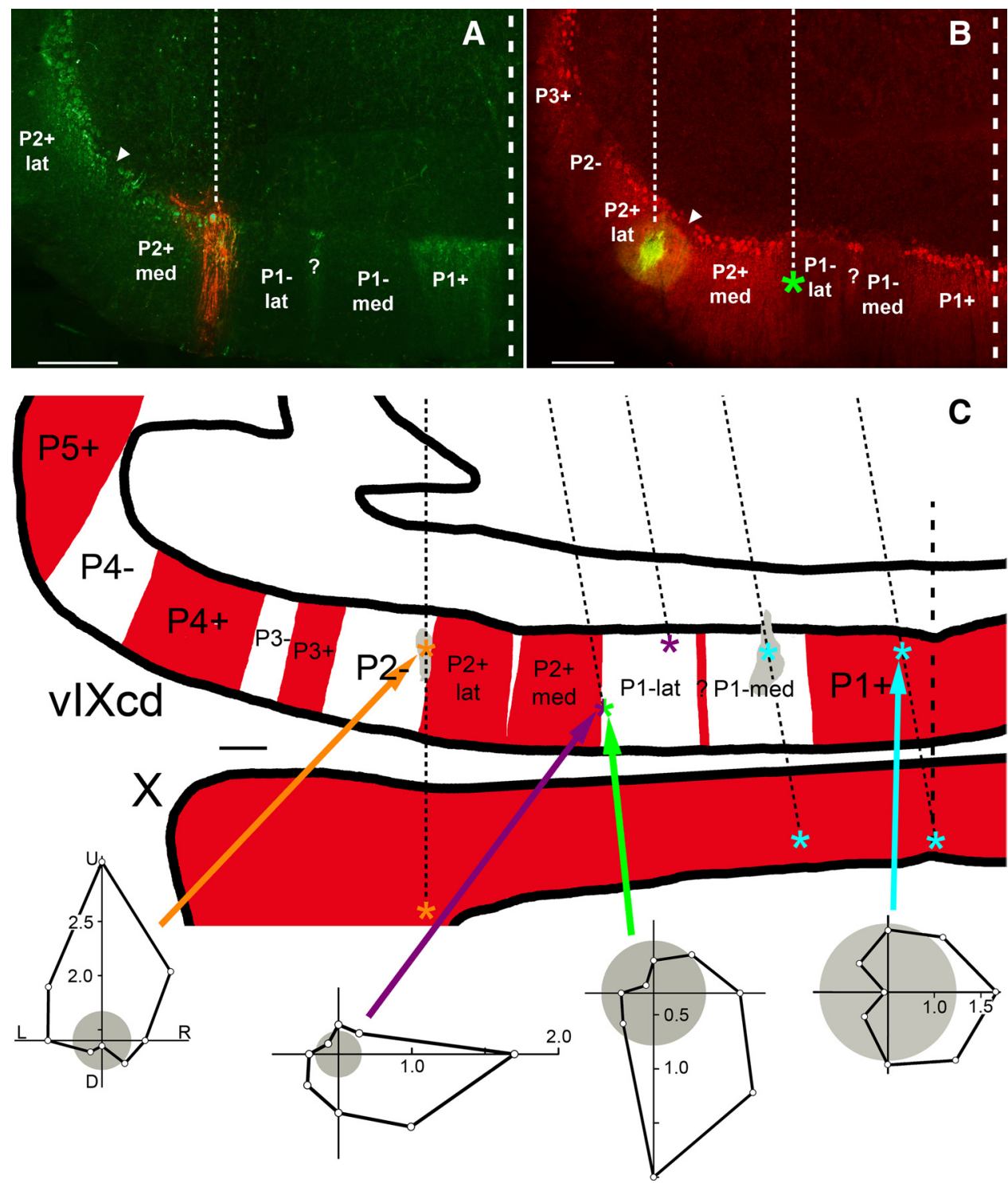

Figure 5. Localizing recording sites to Zll stripes in the ventral uvula. $\boldsymbol{A}$ and $\boldsymbol{B}$ show photomicrographs of a coronal section through the caudal region of folium IXcd. In $\boldsymbol{A}(\boldsymbol{B})$, an injection of red (green) CTB is shown with ZII + labeling in green (red). The thin dashed lines denote the electrode tracks, whereas the thick dashed lines indicate the midline. The inverted triangles indicate the Zll-immunonegative notch separating the medial and lateral portions of $\mathrm{P} 2+(\mathrm{P} 2+$ med, $\mathrm{P} 2+$ lat). In $A$, an expansion cell was recorded at the injection site, located in $\mathrm{P} 2+$ med. In $B$, a descent cell was recorded at the injection site in P2 + lat. In the rightmost track, located $600 \mu \mathrm{m}$ medial to the injection track, an ascent cell was recorded as indicated ${ }^{*}$ ) in the lateral portion of the P1 - stripe (P1 - lat). C shows a drawing of coronal section through folia IXcd and X with ZII + and ZII - stripes indicated, respectively, by red and white shading in the molecular layer. Multiple parallel electrode penetrations are represented by thin dashed lines. The medial-most injection from the oblique electrode penetration (green (TB) was easily distinguished from the lateral-most injection from the vertical electrode penetration (red (TB), and was used as the reference point for reconstructing the remaining oblique electrode penetrations, which were based on stereotaxic coordinates. The orange, purple, and light blue asterisks indicated locations where descent, expansion, and contraction cells were recorded, respectively. At the half-green/half-purple asterisk on the lateral edge of P1 - lat, both ascent and expansion units were recorded at the same location. (The two units were separated with spike sorting.) The two shaded gray areas indicate the locations of two injection sites. Representative direction tuning curves are shown for each type of neuron. Firing rate is plotted as a function of direction of motion in polar coordinates. The gray circles represent the spontaneous rates. Scale bars: $A, B, 300 \mu \mathrm{m} ; C, 250 \mu \mathrm{m}$. U, D, R, L, Upward, downward, rightward, and leftward motion.

MI for cells responsive to visual stimulation was $0.48 \pm 0.017$ (mean \pm SEM; range, $0.2-1.0$ ). Of the 125 recording sites, 18 of these were in folium X ( 6 contraction, 1 ascent, 7 descent, 4 rVA), and 107 were in IXcd ( 13 contraction, 26 expansion, 15 ascent, 41 descent, 6 rVA, $6 \mathrm{NM})$.

\section{ZII immunohistochemistry of folium IXcd}

After ZII expression was visualized in the coronal sections throughout the cerebellum, using immunohistochemical techniques, the locations of the 107 recording sites in IXcd were assigned to a particular ZII stripe. In the cerebella of all animals, we observed the expected pattern of ZII immunoreactivity in folium IXcd (Figs. $1 B, 5$ ) consisting of seven $\mathrm{ZII}+/-$ stripes. We used the nomenclature from Pakan et al. (2007), whereby the most medial positive stripe is designated as $\mathrm{P} 1+$ and the number increases as the stripes move laterally to P7+ (Fig. 1B) (Brochu et al., 1990; Eisenman and Hawkes, 1993; Ozol et al., 1999; Sillitoe and Hawkes, 2002) (for review, see Sillitoe et al., 2005). The width of individual stripes can vary both between animals as well as along the rostrocaudal axis of the cerebellum within animals. Therefore, in designating the stripe numbers, it was important to complete an examination of all sections throughout the rostro- 


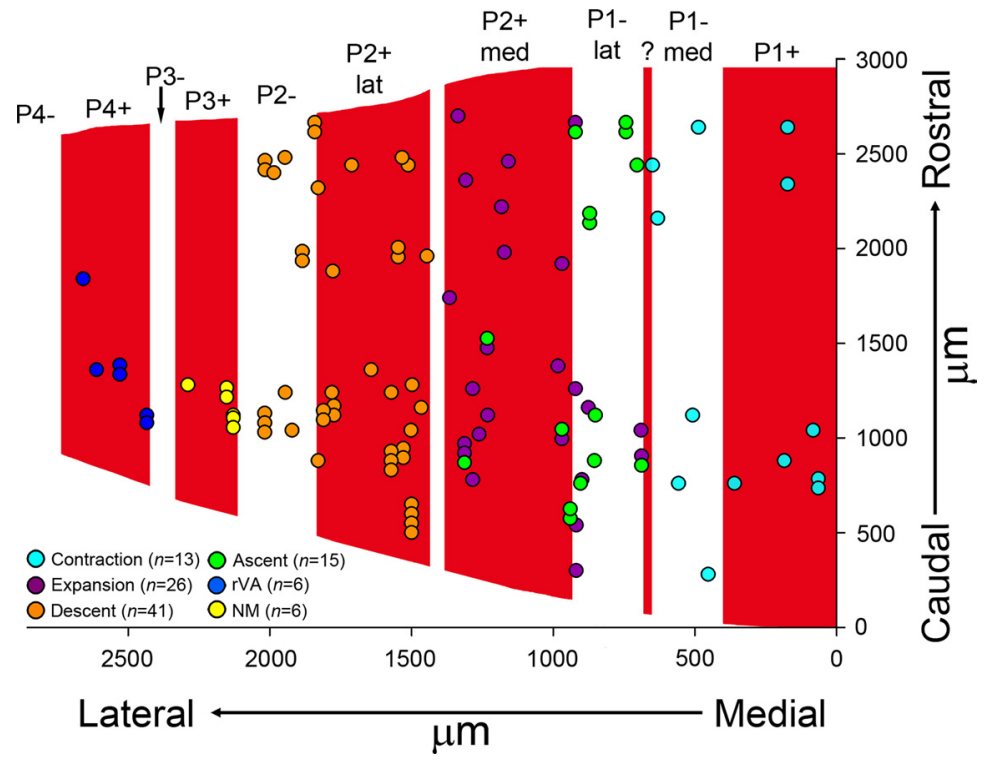

Figure 6. Distributions of optic flow neurons in Zll stripes in the ventral uvula. This figure shows the recording sites of contraction (light blue), expansion (purple), ascent (green), descent (orange); cells not modulated to visual stimuli (yellow) and rVA (dark blue) cells from all cases are indicated. Both the caudorostral ( $y$-axis) and mediolateral ( $x$-axis) position are indicated. For the caudorostral axis, all measurements are relative to the most caudal section containing folium IXcd. For the medial-lateral axis, to permit comparisons between cases, the width of each ZII stripe was normalized (see text for more details). In total, 107 recording sites are indicated. The optic flow zones correspond to the Zll stripes as follows: contraction, P1 + and P1-med; expansion/ ascent, $\mathrm{P} 1-$ lat and $\mathrm{P} 2+$ med; descent, $\mathrm{P} 2+$ lat and $\mathrm{P} 2+$. The six cells that were not modulated (NM) by visual stimuli were all localized to P3 + . Consistent with Pakan et al. (2011), the six rVA cells were localized to P4+.

Table 1. Distribution of contraction, expansion, ascent, descent, nonmodulated to optokinetic stimulation (NM), and rotation about the vertical axis (rVA) cells in zebrin II stripes of folium IXcd in the pigeon cerebellum

Zll stripe

$\mathrm{P} 1+\mathrm{P} 1-$ med $\mathrm{P} 1-$ lat $\mathrm{P} 2+$ med $\mathrm{P} 2+$ lat $\mathrm{P} 2-\mathrm{P} 3+\mathrm{P} 3-\mathrm{P} 4+$

$\begin{array}{lll}\text { Contraction cells } & 7 & 6\end{array}$

Expansion cells

Ascent cells

Descent cells

NM cells

rVA cells

$\begin{array}{rr}10 & 16 \\ 10 & 5\end{array}$

16
5

$28 \quad 13$

6

caudal extent of IXcd. Nonetheless, the seven ZII + / - stripe pairs were easily identifiable. Abutting the midline is a wide ZII + stripe $(\mathrm{P} 1+)$ followed by a wide $\mathrm{ZII}-$ stripe $(\mathrm{P} 1-)$. The $\mathrm{P} 1-$ stripe is separated into medial $(\mathrm{P} 1-$ med) and lateral $(\mathrm{P} 1-$ lat $)$ portions by a thin ZII+ stripe that is one to three PCs wide (see "?" in Figs. $1 B, 5 A, B) . \mathrm{P} 2+$ is consistently wide throughout the rostrocaudal extent of IXcd and is separated into medial $(\mathrm{P} 2+$ med $)$ and lateral (P2+lat) portions by a small "notch" $50 \mu \mathrm{m}$ wide in the middle that appeared to contain no PCs (Pakan et al., 2010) (Figs. $1 B$, $5 A, B$, inverted triangle). The $\mathrm{P} 3+/-$ stripe pair was relatively thin, with the P3 - stripe becoming thinner rostrally. The more lateral stripes, $\mathrm{P} 4+$ to $\mathrm{P} 7-($ Fig. $1 \mathrm{~B}$ ), are part of the flocculus where cells that respond to rotational optic flow are found (Pakan et al., 2011).

The injections were easily identifiable and could be ascribed to a particular ZII stripe. Figure $5 A$ shows an injection of red-CTB, where an expansion unit was recorded. The section was immunoreacted for ZII using a green secondary, and the injection can be seen in P2+med. Figure $5 B$ shows an injection of green-CTB and the section was processed for ZII using a red secondary. A descent cell was recorded with the injection electrode, which was localized to P2+lat. Within the plane of section, there was a second electrode penetration $600 \mu \mathrm{m}$ medial (thin dashed line). An ascent cell was recorded at the location indicated by the green asterisk, located in $\mathrm{P} 1-$ lat.

When the location of all the recording sites in IXcd was localized to a particular ZII stripe, the results were unambiguous: (1) contraction CSA was localized to $\mathrm{P} 1+$ and P1-med, (2) ascent and expansion CSA was localized to $\mathrm{P} 1-$ lat and $\mathrm{P} 2+$ med, and (3) descent cells were localized to P2+lat and $\mathrm{P} 2-$. This is illustrated in one case shown in Figure 5C, in which a drawing of a coronal section through IXcd and $\mathrm{X}$ is shown, with the ZII + stripes indicated in red. The locations of eight recording sites along five electrode penetrations (dashed lines) are shown. The orange, purple, and light blue asterisks indicated locations where descent, expansion, and contraction cells were recorded, respectively. The asterisk that is half-purple/half-green indicates where both an ascent neuron and an expansion neuron were recorded at the same location using a single recording electrode. The two shaded gray areas in the ventral lamella of folium IXcd indicate two separate injection sites. Representative direction tuning curves are shown for each type of neuron. At four sites (one in $\mathrm{P} 1+$, one in $\mathrm{P} 1$ - med, and two in folium $\mathrm{X}$ ), contraction neurons were recorded. At two sites (one in $\mathrm{P} 2-$ and one folium X), descent neurons were recorded. At two sites (both in P1-lat), expansion neurons were recorded and at one site (in P1-lat), an ascent neuron was recorded.

The locations of all 107 recording sites in IXcd from the 24 animals are shown in Figure 6. Because there is variability between animals with respect to the width of the ZII stripes, the data have been collapsed onto an average array of stripes. The ZII stripes shown in Figure 6 have been averaged from measurements of several cases. The medial-lateral location of a data point was normalized to the width of the stripe in which it was found [i.e., from 0 (medial) to $100 \%$ (lateral)] and then scaled to the width of the stripe in Figure 6. For example, the blue circle indicated by the asterisk in Figure 6 represents a recording site in the P1+ stripe, which measured $439 \mu \mathrm{m}$ in width in that section. The recording site was located $189 \mu \mathrm{m}$ from the medial border of the P1+ stripe and thus was normalized to $43 \%$. The width of the average P1+ stripe was $400 \mu \mathrm{m}$, and thus the location was scaled to $172 \mu \mathrm{m}$ lateral to the medial edge of $\mathrm{P} 1+$. For the caudorostral axis, all measurements are relative to the most caudal section containing folium IXcd.

These data are quantified in Table 1 . Of the 13 contraction recordings in IXcd, 7 and 6 cells were localized to ZII stripes P1+ and $\mathrm{P} 1-$ med, respectively. Of the 26 expansion recordings in IXcd, 10 and 16 were localized to ZII stripes P1-lat and $\mathrm{P} 2+$ med, respectively. Of the 15 ascent recordings in IXcd, 10 and 5 cells were localized to ZII stripes P1-lat and P2+med, respectively. Of the 41 ascent recordings in IXcd, 28 and 13 cells were localized to the ZII stripes $\mathrm{P} 2+$ lat and $\mathrm{P} 2-$, respectively. rVA cells were found in the $\mathrm{P} 4+$, confirming results found in previous studies (Pakan et al., 2011). In the P3+ stripe, CSA was 


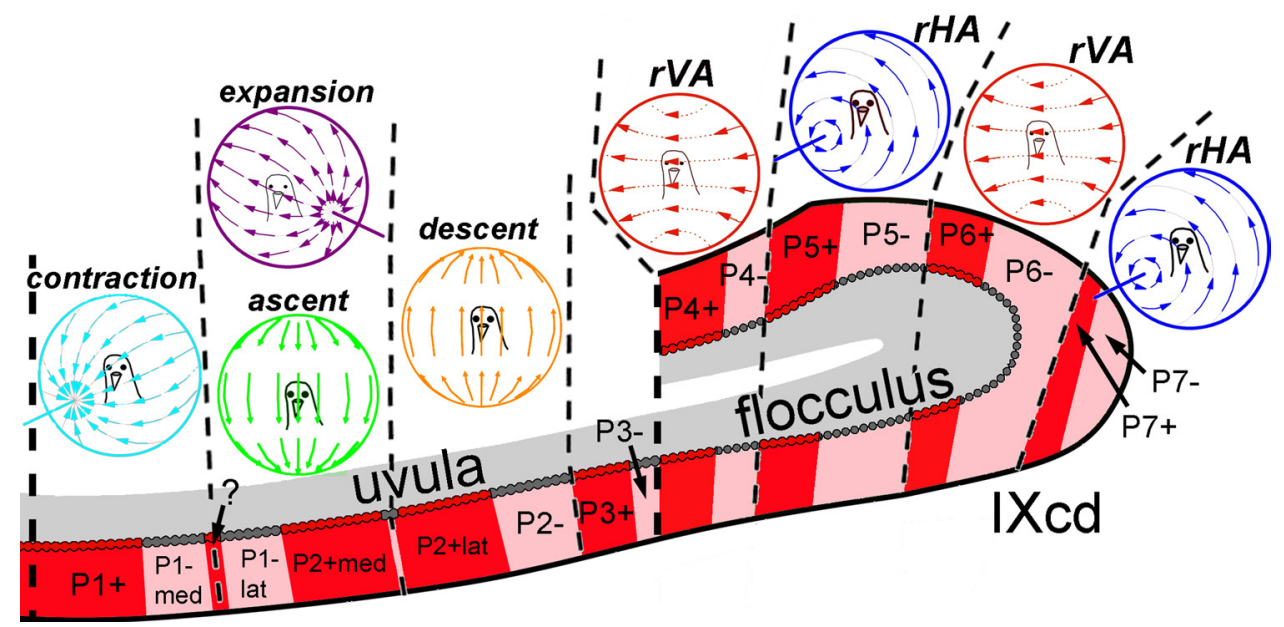

Figure 7. Optic flow neurons correspond with ZII stripes in folium IXcd. ZII-positive PCs are represented as red, whereas ZII-negative PCs are gray and specific zones are indicated from P1 + to P7-. The PCs responsive to contraction (light blue), expansion (purple), ascent (green), descent (orange), rVA (red), and rHA (dark blue) are separated by black dashed lines.

recorded; however, cells in this stripe were not modulated by visual stimulation. Unfortunately, no recordings were obtained from the P3- ZII stripe, which is not surprising as it is so small ( $<100 \mu \mathrm{m}$ wide). The small size of "?" ( $\sim 30 \mu \mathrm{m}$ wide) and the "notch" in $\mathrm{P} 2+(\sim 50 \mu \mathrm{m}$ wide $)$ also made it impossible for us reliably localize recordings to these stripes.

We performed $t$ tests to determine whether there were differences in direction preference between cells localized to the $\mathrm{ZII}+$ stripe versus the ZII - stripe. For the contraction, descent, and ascent cells, there were no significant differences in this regard (all $p>0.05$ ). However, there was a significant difference in the preferred directions for expansion cells in the $\mathrm{P} 1$ - lat stripe compared with those in the $\mathrm{P} 2+$ med stripe $(p<$ $0.007)$. The mean preferred directions for the expansion cells in the P1-lat stripe and $\mathrm{P} 2+$ med stripe were 104 and $87^{\circ}$, respectively $\left(0^{\circ}=\right.$ up) $($ Fig. $4 H)$.

\section{Discussion}

In the present study, we have shown that a given $\mathrm{ZII}+/-$ stripe pair in the ventral uvula corresponds to the specific optic flow preference of PCs within that ZII stripe (Table 1; Figs. 6, 7). We found that (1) contraction cells are localized to the P1+ and P1-med stripes, (2) expansion and ascent cells are localized to $\mathrm{P} 1$ - lat and P2 + med stripes, and (3) descent cells are localized to the $\mathrm{P} 2+$ lat and $\mathrm{P} 2-$ stripes.

There are three points that we need to emphasize. First, a functional unit in the ventral uvula spans a single ZII $+/-$ pair. This relationship between ZII and functional zones was previously shown in the flocculus by Pakan et al. (2011), where they found that rVA zones were confined to $\mathrm{P} 4+/-$ and $\mathrm{P} 6+/-$, whereas rHA zones were confined to $\mathrm{P} 5+/-$ and $\mathrm{P} 7+/-$. Thus, $\mathrm{ZII}$ is related to $\mathrm{PC}$ response properties across the entire folium IXcd.

Second, the present study showed that satellite bands and subzones found in the ventral uvula are not merely superfluous, as they delineate electrophysiological borders. Pakan et al. (2007) remarked a small immunopositive band in the middle of P1(called "?") separating it into P1-med and P1-lat in folium IXcd. They also described a natural paucity of PCs in the middle of $\mathrm{P} 2+$ dividing it into $\mathrm{P} 2+$ med and $\mathrm{P} 2+$ lat. Small satellite bands, or subzones, such as this can also been seen in the cerebella of mammals (Hawkes and Leclerc, 1987; Sanchez et al., 2002). As shown in Figure 6, "?" clearly defines the border between the location of contraction cells in P1-med, and expansion and ascent cells in $\mathrm{P} 1$ - lat. The "notch" in the middle of $\mathrm{P} 2+$ provides the boundary between cells responsive to expansion and ascent cells in P2+med, and descent cells in P2+lat. The optic flow preferences of PCs in the "?" satellite zone are unknown and beyond the sensitivity of our methods as we were not able to reliably localize a recording site to this stripe given its small size (30 $\mu \mathrm{m})$.

Third, the organization revealed in the ventral uvula was not exactly as expected. Within the ventral uvula, our laboratory has previously grouped the few ascent cells that we recorded into the most lateral functional zone along with descent cells (Fig. 1A) (Wylie and Frost, 1999). However, the present study showed that ascent cells were colocalized with expansion cells in the zone medial to the descent cells (Fig. 6) in ZII zones P1-lat and $\mathrm{P} 2+$ med. Furthermore, we were not able to subdivide the expansion and ascent zones, and even recorded both cell types at a single location (for details, see Fig. 3). We have no explanation as to why these two cell types coexist in the same functional zone. The flocculus has a seemingly opposite conundrum as there are multiple zones with the same function (e.g., two rVA and two rHA zones) (Voogd and Wylie, 2004). One possible explanation is that gaze stabilization to rotational optic flow can be achieved with either compensatory head or eye movements. Perhaps one zone is mainly involved in head movements, and the other in eye movements. Finally, we also found that PCs in ZII zones P3+/were not responsive to visual motion stimulation. This could be related to the $\mathrm{C} 2$ zone found in the flocculus of mammals, which is nonresponsive to optokinetic stimulation, and is proposed to be involved in generating head movements (De Zeeuw and Koekkoek, 1997). It could also be a vestibular zone, similar to those described in the uvula/nodulus of rabbits by Barmack and Shojaku (1995).

\section{Relationship of zebrin II immunoreactivity to function in folium IXcd}

Figure 7 shows the organization of PCs responsive to different types of optic flow in relation to ZII stripes. We want to emphasize that a physiological zone in folium IXcd spans a single $\mathrm{ZII}+/-$ band. Although the present study and that of Pakan et al. (2011) found that CSA of PCs within a given optic flow zone was identical in both the ZII+ and ZII - stripe, recent evidence suggests that ZII+ and ZII - stripes are functionally different. For 
example, Pakan et al. (2010) showed that MF inputs from two retinorecipient nuclei in pigeons that are responsive to optic flow, the pretectal nucleus lentiformis mesencephali (LM) and the nucleus of the basal optic root (nBOR), project preferentially to $\mathrm{ZII}+$ stripes in IXcd. Whether there are MF afferents that project preferentially to ZII- PCs in the pigeon remains to be seen.

It has recently been suggested that PCs in the ZII+ and ZIIstripes within an optic flow zone have differential projections to the vestibular and cerebellar nuclei (Sugihara, 2011; Wylie et al., 2012) as axon terminals from ZII + and ZII- PCs are found in different areas of these nuclei. However, the functional difference between ZII+ and ZII- PCs remains unclear, although several studies have pointed to the different roles ZII+ and ZII- PCs might play in cerebellar physiology (Nagao et al., 1997; Wadiche and Jahr, 2005; Sugihara et al., 2007; Ebner et al., 2012). Specifically, Paukert et al. (2010) recently showed that climbing fibers in $\mathrm{ZII}+$ stripes release more glutamate per action potential than climbing fibers in ZII - stripes and proposed that ZII + PCs undergo more activity-dependent synaptic plasticity than ZIIPCs. This suggests that a corticonuclear system originating in ZII+ PCs that is more plastic runs in parallel with a corticonuclear system originating in ZII - PCs that is not as plastic and may receive different mossy fiber inputs.

\section{The utility of the vestibulocerebellum as a general model for cerebellar organization}

The pigeon $\mathrm{VbC}$ provides an excellent model for cerebellar organization. The flocculus is functionally identical in mammals and birds, consisting of rVA and rHA cells that are organized into multiple parasagittal zones (for review, see Voogd and Wylie, 2004). Unlike pigeons, the flocculus is uniformly zebrin positive in rodents (Hawkes and Herrup, 1995). This is not the case for all mammals, as the marmoset flocculus contains four alternating ZII+/- stripe pairs (Fujita et al., 2010). Whether the rVA and rHA zones seen in the marmoset also span a ZII $+/-$ stripe, as in birds, has yet to be determined. The uvula/nodulus in birds and mammals is functionally similar insofar as it is involved in selftranslation (Wylie et al., 1993; Barmack and Shojaku, 1995; Yakusheva et al., 2008). In both birds and mammals, the nodulus is uniformly ZII+, while the uvula contains a number of alternating ZII $+/-$ stripes in all species investigated (Hawkes and Herrup, 1995; Pakan et al., 2007; Marzban and Hawkes, 2011). Data suggest that the ZII stripes in the uvula are not organized in rodents as we have shown in the present study. Voogd and Ruigrok (1997) showed that the ZII - stripes in the rodent vermis likely receive trigeminal input. Moreover, Sugihara and Shinoda (2004) showed that the ZII - and ZII + stripes in the rodent uvula receive climbing fiber input from quite different olivary regions. As such, it is unlikely that the CSA in adjacent $+/-$ pairs responds to the same type of stimuli.

Thus, while our findings certainly contribute to general principles of cerebellar organization, much more is needed to determine how multisensory information is processed throughout the cerebellum. For example, while the vestibular nuclei, spinal cord, and pontine nuclei also send MF afferents to IXcd (Freedman et al., 1975; Clarke, 1977; Vielvoye and Voogd, 1977; Necker, 1992; Pakan and Wylie, 2008), their relationship to ZII has not been investigated. Do some of these inputs preferentially target the granular layer subjacent to either ZII + or ZII- PCs in IXcd? We have previously shown that PCs in VA and HA zones have several non-overlapping projections to different areas in the vestibular and cerebellar nuclei (Wylie et al., 2003a,b). Do the PCs in the ZII + and ZII- stripes within a physiological zone have differen- tial projections? As further research will help answer these and other questions, the pigeon $\mathrm{VbC}$ continues to emerge as a model system in which to elucidate the general principles of cerebellar organization.

\section{Zebrin and functional units throughout the cerebellum}

Our major point is that a ZII $+/-$ stripe pair represents a functional unit in the cerebellum. As most the cerebellum consists of alternating ZII + and ZII - stripes, we hypothesize that this relationship applies as a general rule. However, this clearly cannot apply to areas of the cerebellum where ZII is uniformly immunopositive. In both birds and mammals, this includes folia $\mathrm{X}$ and VI/VII (Hawkes and Herrup, 1995; Pakan et al., 2007). Functional zones are clearly apparent in these areas based on climbing fiber afferents and PC response properties (Voogd and Bigare, 1980; Barmack and Shojaku, 1995). In folium X of pigeons, the optokinetic zones extend from those in IXcd (i.e., in IXcd, the functional zones span a ZII+/- stripe pair, but in X, all the PCs are ZII immunopositive). It is possible that the zones in folia that are uniformly ZII+ are delineated by other molecular markers. For example, heat shock protein 25 is expressed in stripes in folia VI/VII and X in mammals (Armstrong et al., 2000; Schonewille et al., 2006).

\section{References}

Ahn AH, Dziennis S, Hawkes R, Herrup K (1994) The cloning of zebrin II reveals its identity with aldolase C. Development 120:2081-2090.

Andersson G, Oscarsson O (1978) Climbing fiber microzones in cerebellar vermis and their projection to different groups of cells in the lateral vestibular nucleus. Exp Brain Res 32:565-579.

Armstrong CL, Krueger-Naug AM, Currie RW, Hawkes R (2000) Constitutive expression of the $25 \mathrm{kDa}$ heat shock protein Hsp25 reveals novel parasagittal bands of Purkinje cells in the adult mouse cerebellar cortex. J Comp Neurol 416:383-397.

Barmack NH, Shojaku H (1995) Vestibular and visual climbing fiber signals evoked in the uvula-nodulus of the rabbit cerebellum by natural stimulation. J Neurophysiol 74:2573-2589.

Blasdel GG, Salama G (1986) Voltage-sensitive dyes reveal a modular organization in monkey striate cortex. Nature 321:579-585.

Bloedel JR, Roberts WJ (1971) Action of climbing fibers in cerebellar cortex of the cat. J Neurophysiol 34:17-31.

Brochu G, Maler L, Hawkes R (1990) Zebrin II: a polypeptide antigen expressed selectively by Purkinje cells reveals compartments in rat and fish cerebellum. J Comp Neurol 291:538-552.

Clarke PG (1977) Some visual and other connections to the cerebellum of the pigeon. J Comp Neurol 174:535-552.

De Zeeuw CI, Koekkoek SK (1997) Signal processing in the C2 module of the flocculus and its role in head movement control. Prog Brain Res 114:299-320.

De Zeeuw CI, Wylie DR, DiGiorgi PL, Simpson JI (1994) Projections of individual Purkinje cells of identified zones in the flocculus to the vestibular and cerebellar nuclei in the rabbit. J Comp Neurol 349:428-447.

Ebner TJ, Wang X, Gao W, Cramer SW, Chen G (2012) Parasagittal zones in the cerebellar cortex differ in excitability, information processing, and synaptic plasticity. Cerebellum 11:418-419.

Eisenman LM, Hawkes R (1993) Antigenic compartmentation in the mouse cerebellar cortex: zebrin and HNK-1 reveal a complex, overlapping molecular topography. J Comp Neurol 335:586-605.

Ekerot CF, Larson B (1973) Correlation between sagittal projection zones of climbing and mossy fiber paths in cat cerebellar anterior lobe. Brain Res 64:446-450.

Felleman DJ, Van Essen DC (1991) Distributed hierarchical processing in the primate cerebral cortex. Cereb Cortex 1:1-47.

Freedman SL, Feirabend HK, Vielvoye GJ, Voogd J (1975) Re-examination of the ponto-cerebellar projection in the adult white leghorn (Gallus domesticus). Acta Morphol Neerl Scand 13:236-238.

Fujita H, Oh-Nishi A, Obayashi S, Sugihara I (2010) Organization of the marmoset cerebellum in three-dimensional space: lobulation, aldolase $\mathrm{C}$ 
compartmentalization and axonal projection. J Comp Neurol 518:1764-1791.

Gao W, Chen G, Reinert KC, Ebner TJ (2006) Cerebellar cortical molecular layer inhibition is organized in parasagittal zones. J Neurosci 26:8377-8387.

Goldman-Rakic PS (1984) Modular organization of prefrontal cortex. Trends Neurosci 7:419-424.

Goodale MA, Milner AD (1992) Separate pathways for perception and action. Trends Neurosci 15:20-25.

Graf W, Simpson JI, Leonard CS (1988) Spatial organization of visual messages of the rabbit's cerebellar flocculus. II. Complex and simple spike responses of Purkinje cells. J Neurophysiol 60:2091-2121.

Granit R, Phillips CG (1956) Excitatory and inhibitory processes acting upon individual Purkinje cells of the cerebellum in cats. J Physiol 133:520-547.

Hawkes R (1992) Antigenic markers of cerebellar modules in the adult mouse. Biochem Soc Trans 20:391-395.

Hawkes R, Gravel C (1991) The modular cerebellum. Prog Neurobiol 36:309-327.

Hawkes R, Herrup K (1995) Aldolase C/zebrin II and the regionalization of the cerebellum. J Mol Neurosci 6:147-158.

Hawkes R, Leclerc N (1987) Antigenic map of the rat cerebellar cortex: the distribution of parasagittal bands as revealed by monoclonal antiPurkinje cell antibody mabQ113. J Comp Neurol 256:29-41.

Herculano-Houzel S (2010) Coordinated scaling of cortical and cerebellar numbers of neurons. Front Neuroanat 4:12.

Hubel DH, Wiesel TN (1962) Receptive fields, binocular interaction and functional architecture in the cat's visual cortex. J Physiol 160:106-154

Iwaniuk AN, Marzban H, Pakan JM, Watanabe M, Hawkes R, Wylie DR (2009) Compartmentation of the cerebellar cortex of hummingbirds (Aves: Trochilidae) revealed by the expression of zebrin II and phospholipase C $\beta 4$. J Chem Neuroanat 37:55-63.

Karten H, Hodos W (1967) A stereotaxic atlas of the brain of the pigeon (Columba livia). Baltimore: Johns Hopkins.

Llinás R, Sasaki K (1989) The functional organization of the olivo-cerebellar system as examined by multiple Purkinje cell recordings. Eur J Neurosci $1: 587-602$.

Marzban H, Hawkes R (2011) On the architecture of the posterior zone of the cerebellum. Cerebellum 10:422-434.

Mostofi A, Holtzman T, Grout AS, Yeo CH, Edgley SA (2010) Electrophysiological localization of eyeblink-related microzones in rabbit cerebellar cortex. J Neurosci 30:8920-8934.

Mountcastle VB (1997) The columnar organization of the neocortex. Brain 120:701-722.

Nagao S, Kitazawa H, Osanai R, Hiramatsu T (1997) Acute effects of tetrahydrobiopterin on the dynamic characteristics and adaptability of vestibulo-ocular reflex in normal and flocculus lesioned rabbits. Neurosci Lett 231:41-44.

Necker R (1992) Spinal neurons projecting to anterior or posterior cerebellum in the pigeon. Anat Embryol (Berl) 185:325-334.

Ozol K, Hayden JM, Oberdick J, Hawkes R (1999) Transverse zones in the vermis of the mouse cerebellum. J Comp Neurol 412:95-111.

Pakan JM, Wylie DR (2008) Congruence of zebrin II expression and functional zones defined by climbing fiber topography in the flocculus. Neuroscience 157:57-69.

Pakan JM, Iwaniuk AN, Wylie DR, Hawkes R, Marzban H (2007) Purkinje cell compartmentation as revealed by zebrin II expression in the cerebellar cortex of pigeons (Columba livia). J Comp Neurol 501:619-630.

Pakan JM, Graham DJ, Wylie DR (2010) Organization of visual mossy fiber projections and zebrin expression in the pigeon vestibulocerebellum. J Comp Neurol 518:175-198.

Pakan JM, Graham DJ, Gutiérrez-Ibáñez C, Wylie DR (2011) Organization of the cerebellum: correlating zebrin immunochemistry with optic flow zones in the pigeon flocculus. Vis Neurosci 28:163-174.

Paukert M, Huang YH, Tanaka K, Rothstein JD, Bergles DE (2010) Zones of enhanced glutamate release from climbing fibers in the mammalian cerebellum. J Neurosci 30:7290-7299.

Ruigrok TJ (2003) Collateralization of climbing and mossy fibers projecting to the nodulus and flocculus of the rat cerebellum. J Comp Neurol $466: 278-298$.

Sanchez M, Sillitoe RV, Attwell PJ, Ivarsson M, Rahman S, Yeo CH, Hawkes R
(2002) Compartmentation of the rabbit cerebellar cortex. J Comp Neurol 444:159-173

Sato Y, Miura A, Fushiki H, Kawasaki T (1993) Barbiturate depresses simple spike activity of cerebellar Purkinje cells after climbing fiber input. J Neurophysiol 69:1082-1090.

Schonewille M, Luo C, Ruigrok TJ, Voogd J, Schmolesky MT, Rutteman M, Hoebeek FE, De Jeu MT, De Zeeuw CI (2006) Zonal organization of the mouse flocculus: physiology, input, and output. J Comp Neurol 497:670-682.

Sillitoe RV, Hawkes R (2002) Whole-mount immunohistochemistry: a high-throughput screen for patterning defects in the mouse cerebellum. J Histochem Cytochem 50:235-244.

Sillitoe RV, Marzban H, Larouche M, Zahedi S, Affanni J, Hawkes R (2005) Conservation of the architecture of the anterior lobe vermis of the cerebellum across mammalian species. Prog Brain Res 148:283-297.

Sugihara I (2011) Compartmentalization of the deep cerebellar nuclei based on afferent projections and aldolase C expression. Cerebellum 10: $449-463$.

Sugihara I, Quy PN (2007) Identification of aldolase C compartments in the mouse cerebellar cortex by olivocerebellar labeling. J Comp Neurol 500:1076-1092.

Sugihara I, Shinoda Y (2004) Molecular, topographic, and functional organization of the cerebellar cortex: a study with combined aldolase $\mathrm{C}$ and olivocerebellar labeling. J Neurosci 24:8771-8785.

Sugihara I, Shinoda Y (2007) Molecular, topographic, and functional organization of the cerebellar nuclei: analysis by three-dimensional mapping of the olivonuclear projection and aldolase C labeling. J Neurosci 27:9696-9710.

Sugihara I, Marshall SP, Lang EJ (2007) Relationship of complex spike synchrony bands and climbing fiber projection determined by reference to aldolase C compartments in crus IIa of the rat cerebellar cortex. J Comp Neurol 501:13-29.

Ungerleider LG, Mishkin M (1982) Two cortical visual systems. In: Analysis of visual behavior (Ingle DJ, Goodale MA, Mansfield RJW, eds) pp 549_ 585. Cambridge, MA: MIT

Vielvoye GJ, Voogd J (1977) Time dependence of terminal degeneration in spino-cerebellar mossy fiber rosettes in the chicken and the application of terminal degeneration in successive degeneration experiments. J Comp Neurol 175:233-242.

Voogd J (1967) Comparative aspects of the structure and fiber connections of the mammalian cerebellum. Prog Brain Res 25:94-134.

Voogd J, Bigare F (1980) Topographical distribution of olivary and corticonuclear fibers in the cerebellum: a review. In: The olivary nucleus. Anatomy and physiology (de Montigny C, Courville J, eds), pp 207-234. New York: Raven.

Voogd J, Ruigrok TJ (1997) Transverse and longitudinal patterns in the mammalian cerebellum. Prog Brain Res 114:21-37.

Voogd J, Ruigrok TJ (2004) The organization of the corticonuclear and olivocerebellar climbing fiber projections to the rat cerebellar vermis: the congruence of projection zones and the zebrin pattern. J Neurocytol 33:5-21.

Voogd J, Wylie DR (2004) Functional and anatomical organization of floccular zones: a preserved feature in vertebrates. J Comp Neurol 470:107-112.

Voogd J, Pardoe J, Ruigrok TJ, Apps R (2003) The distribution of climbing and mossy fiber collateral branches from the copula pyramidis and the paramedian lobule: congruence of climbing fibercortical zones and the pattern of zebrin banding within the rat cerebellum. J Neurosci 23:4645-4656.

Wadiche JI, Jahr CE (2005) Patterned expression of Purkinje cell glutamate transporters controls synaptic plasticity. Nat Neurosci 8:1329-1334.

Winship IR, Wylie DR (2001) Responses of neurons in the medial column of the inferior olive in pigeons to translational and rotational optic flowfields. Exp Brain Res 141:63-78.

Winship IR, Wylie DR (2003) Zonal organization of the vestibulocerebellum in pigeons (Columba livia): I. Climbing fiber input to the flocculus. J Comp Neurol 456:127-139.

Winship IR, Wylie DR (2006) Receptive-field structure of optic flow responsive Purkinje cells in the vestibulocerebellum of pigeons. Vis Neurosci 23:115-126.

Wu HS, Sugihara I, Shinoda Y (1999) Projection patterns of single mossy 
fibers originating from the lateral reticular nucleus in the rat cerebellar cortex and nuclei. J Comp Neurol 411:97-118.

Wylie DR, Frost BJ (1993) Responses of pigeon vestibulocerebellar neurons to optokinetic stimulation. II. The 3-dimensional reference frame of rotation neurons in the flocculus. J Neurophysiol 70:2647-2659.

Wylie DR, Frost BJ (1999) Complex spike activity of Purkinje cells in the ventral uvula and nodulus of pigeons in response to translational optic flow. J Neurophysiol 81:256-266.

Wylie DR, Kripalani T, Frost BJ (1993) Responses of pigeon vestibulocerebellar neurons to optokinetic stimulation. I. Functional organization of neurons discriminating between translational and rotational visual flow. J Neurophysiol 70:2632-2646.

Wylie DR, Brown MR, Barkley RR, Winship IR, Crowder NA, Todd KG (2003a) Zonal organization of the vestibulocerebellum in pigeons (Columba livia): II. Projections of the rotation zones of the flocculus. J Comp Neurol 456:140-153.

Wylie DR, Brown MR, Winship IR, Crowder NA, Todd KG (2003b) Zonal organization of the vestibulocerebellum in pigeons (Columba livia): III. Projections of the translation zones of the ventral uvula and nodulus. J Comp Neurol 465:179-194.

Wylie DR, Pakan JM, Huynh H, Graham DJ, Iwaniuk AN (2012) Distribution of zebrin-immunoreactive Purkinje cell terminals in the cerebellar and vestibular nuclei of birds. J Comp Neurol 520:1532-1546.

Yakusheva T, Blazquez PM, Angelaki DE (2008) Frequency-selective coding of translation and tilt in macaque cerebellar nodulus and uvula. J Neurosci 28:9997-10009. 